\title{
Fungos Associados com o Declínio e Morte de Videiras no Estado do Rio Grande do Sul
}

\author{
Lucas da R. Garrido, Olavo R. Sônego \& Vanderlei N. Gomes \\ Laboratório de Fitopatologia, Embrapa Uva e Vinho, Cx. Postal 130, CEP 95700-000, Bento Gonçalves, RS, \\ e-mail: garrido@cnpuv.embrapa.br
}

(Aceito para publicação 24/09/2003)

Autor para correspondência: Lucas da R. Garrido

GARRIDO, L.R., SÔNEGO, O.R \& GOMES, V.N. Fungos associados com o declínio e morte de videiras no Estado do Rio Grande do Sul. Fitopatologia Brasileira 29:322-324. 2004.

\begin{abstract}
RESUMO
Diversas são as causas bióticas e abióticas responsáveis pelo declínio e morte de videiras (Vitis spp.) no Rio Grande do Sul. Dentro do primeiro grupo temos vários fungos fitopatogênicos. O objetivo do trabalho foi levantar as principais espécies de fungos presentes em videiras com os sintomas desta moléstia em vinhedos da Serra Gaúcha. A partir de 107 amostras coletadas em diferentes cultivares e municípios, observou-se uma maior incidência de declínio nas

cultivares de uvas americanas, Vitis labrusca (Bordô, Concord e Niágara), do que nas cultivares de uvas européias, V. vinifera. As principais espécies de fungos encontradas foram: Cylindrocarpon sp., Phaeoacremonium sp., Verticillium sp., Botryosphaeria sp., Fusarium oxysporum f.sp. herbemontis, Graphium sp. e Cylindrocladium sp.

Palavras-chave adicionais: Vitis labrusca, V. vinifera, Cylindrocarpon sp., uva americana.
\end{abstract}

\section{ABSTRACT}

Fungi associated with grapevine showing decline and plant death in the state of Rio Grande do Sul, Southern Brazil

Grapevine (Vitis spp.) decline and death in Rio Grande do Sul can result from biotic and abiotic causes. Several plant pathogenic fungi cause the first group. The objective of this research was to report the main fungal species associated with plants showing symptoms of decline and death in the "Serra Gaúcha" region, Southern
Brazil. The incidence of disfunction in 107 samples from different cultivars and growing regions was higher in American cultivars of $V$. labrusca (Bordô, Concord and Niagara), than in European grapes (Vitis vinifera). The species of fungi found were: Cylindrocarpon sp., Phaeoacremonium sp., Verticillium sp., Botryosphaeria sp., Fusarium oxysporum f.sp. herbemontis, Graphium sp. and Cylindrocladium sp.
O Rio Grande do Sul é responsável por mais de 95\% da produção brasileira de vinhos e derivados, envolvendo cerca de 16.000 produtores e 500 estabelecimentos vinícolas (Mello, 2001). Os vinhos finos brasileiros são produzidos a partir de uvas (Vitis vinifera L.) enquanto os vinhos de mesa são produzidos de V. labrusca $\mathrm{L}$. A videira está sujeita ao ataque de diversas doenças e pragas, as quais reduzem a quantidade e a qualidade da uva produzida, e em muitos casos podem inviabilizar a cultura.

Diversas são as causas que podem levar a videira à morte. Tanto as pragas quanto as doenças quando não controladas adequadamente ocasionam a debilitação progressiva da planta. O número de casos de declínio e morte de plantas de videira tem aumentado de forma acentuada nos últimos anos, causando grande redução de produtividade e de qualidade da uva. Segundo Kuhn (1981), os principais agentes causais identificados na região associados a este problema são: pérola-da-terra (Eurhizococcus brasiliensis Hempel), filoxera (Daktulosphaira vitifoliae Fitch), as cochonilhas-do-tronco (Hemiberlesia lataniae Signoret, Duplaspidiotus tesseratus Charmoy e D. fossor Newstead), viroses que causam o complexo rugoso da videira (intumescimento dos ramos e caneluras do tronco da videira), fusariose (Fusarium oxysporum Schl. f.sp. herbemontis
Tocchetto), fungos que causam podridão radicular [Verticillium sp., Rosellinia necatrix Prill., Armillaria mellea (Vahl:Fr.) P. Kumm e Phytophthora sp.], os fungos que causam apodrecimento do tronco e ramos [Botryosphaeria sp., Eutypa lata (Pers.: fr.) Tul. \& C. Tul., Phomopsis viticola (Sacc.) Sacc. e Stereum sp.]. Devido a complexidade do agroecossistema, para o controle destes agentes devem ser contemplados aspectos de controle integrado, ou seja, medidas que enfoquem a multidisciplinaridade (Garrido \& Sônego, 1999; Pearson \& Goheen, 1988; Sônego et al., 2001) .

Além destes organismos, causas abióticas podem estar associadas ao declínio e morte de plantas de videira como a má soldadura do enxerto, o excesso de umidade no solo, a fitotoxicidade causada por herbicidas ou as misturas de produtos no tanque do pulverizador e por adubações desequilibradas.

$\mathrm{O}$ objetivo do trabalho foi isolar e identificar fungos associados ao declínio e morte de videiras, de vinhedos da região da Serra Gaúcha.

As áreas com histórico de declínio e morte de plantas foram pré-definidas pelos técnicos da extensão rural. Cento e sete amostras de videira das cultivares Bordô, Isabel, Niágara, Concord, Couderc, Dona Zilá, Moscato-Embrapa, Seibel, Tardia de Caxias, Vênus, Cabernet Franc, Cabernet Sauvignon, 
Fungos associados com o declínio e morte de videiras...

Moscato-Yalo, Perlona, Riesling Itálico, Tannat e Trebbiano foram coletadas em 15 municípios da Serra Gaúcha (Antônio Prado, Bento Gonçalves, Caxias do Sul, Flores da Cunha, Farroupilha, Nova Roma do Sul, Nova Pádua, Dois Lajeados, Nova Prata, Pinto Bandeira, Monte Belo do Sul, São Marcos, Feliz, Veranópolis e Garibaldi) em novembro de 2001. Durante o diagnóstico local, foi verificada a presença ou não de pragas, de sintomas típicos de viroses, deficiências nutricionais e de doenças fúngicas da parte aérea e raiz. Logo após, cada amostra (tronco, ramos, folhas e raiz) foi identificada e colocada em saco plástico até a chegada ao Laboratório de Fitopatologia da Embrapa Uva e Vinho.

Partes dos troncos e das raízes foram cortados e colocados em câmara-úmida durante sete dias. Após este período, as amostras foram observadas sob microscópio estereoscópio com aumento de 50 x e em microscópio óptico com aumento de $800 \mathrm{x}$.

$\mathrm{Na}$ ausência de estruturas reprodutivas, porções dos fungos encontrados foram isolados para placas de Petri contendo meio batata-dextrose-ágar (BDA) acidificado com ácido lático, incubados durante 15 dias a temperatura ambiente e fotoperíodo de $12 \mathrm{~h}$ com luz fluorescente.

A identificação dos gêneros dos fungos encontrados foi realizada com auxílio de chaves de identificação (Ellis, 1971; Barnett \& Hunter, 1972; Carmichael et al., 1980; Hanlin, 1990).

A grande maioria das plantas com sintomas de declínio e morte de videiras pertenciam às cultivares americanas: Bordô, Niágara, Isabel e Concord, representando cerca de 78,50\% das amostras coletadas (Tabela 1). Nestas cultivares, verificou-se principalmente a ocorrência de Cylindrocarpon sp. (18,69\%),
Verticillium sp. (17,75\%), Cylindrocladium sp. (5,60\%) e Graphium sp. (16,82\%). A primeira espécie tem sido relatada em países da Europa, Estados Unidos e no Chile como agente causal do "pé-preto" da videira (Grasso \& Magnano di San Lio, 1975; Scheck et al., 1998; Auger et al., 2001), enquanto Verticillium sp. é causador de murchas vasculares em diversas plantas hospedeiras. Além destes, observou-se que é bastante comum encontrar Graphium sp. em troncos de plantas de videira apresentando apodrecimento de madeira. Até o momento, não foram realizados ensaios para certificação do envolvimento do Graphium sp. no declínio das plantas, podendo ser saprófita e não parasita nestes materiais.

Nestes últimos anos vem aumentando, na região, a incidência de podridão descendente causada pelos fungos Botryosphaeria sp. e E. lata (Sônego et al., 1999). Nas avaliações deste trabalho observou-se em aproximadamente 13,08\% das amostras coletadas a presença de Botryosphaeria sp., sendo a maior ocorrência em 'Niágara'. Por ser uma doença de lento desenvolvimento, os viticultores não costumam dar-lhe grande importância. Os sintomas começam a ser observados dois a três anos após infecção e de um modo geral a partir dos ferimentos da poda.

Nas cultivares de uvas americanas coletadas, observouse em $12,14 \%$ das amostras a presença de Phaeoacremonium sp. e em $1,86 \%$ nas cultivares viníferas. Na cultivar 'Niágara' foi encontrada a maior ocorrência. Nestes últimos dez anos, diversos trabalhos têm relatado o envolvimento de Phaeoacremonium chlamydosporum W. Gams et al. com o declínio de videiras na Europa. É reconhecido também por alguns autores, que espécies de Phaeoacremonium que colo-

TABELA 1 - Incidência de fungos associados a diversas cultivares de videiras (Vitis spp.) plantadas na Serra Gaúcha, RS, apresentando sintomas de declínio e isolados da parte aérea e sistema radicular

\begin{tabular}{|c|c|c|c|c|c|c|c|c|}
\hline \multirow{3}{*}{$\begin{array}{l}\text { Cultivar } \\
\text { de videira }\end{array}$} & \multirow{3}{*}{$\begin{array}{l}\text { Número } \\
\text { total de } \\
\text { amostras }\end{array}$} & \multicolumn{7}{|c|}{ Încidência de fungos nas amostras (\%) } \\
\hline & & \multicolumn{3}{|c|}{ Parte aérea } & \multicolumn{4}{|c|}{ Sistema radicular } \\
\hline & & $\begin{array}{l}\text { Botryosphaeria } \\
\text { sp. }\end{array}$ & $\begin{array}{l}\text { Graphium } \\
\text { sp. }\end{array}$ & $\begin{array}{l}\text { Phaeoacremonium } \\
\text { sp. }\end{array}$ & $\begin{array}{c}\text { Fusarium oxysporum } \\
\text { f.sp. herbemontis }\end{array}$ & $\begin{array}{c}\text { Cylindrocarpon } \\
\text { sp. }\end{array}$ & $\begin{array}{c}\text { Verticillium } \\
\text { sp. }\end{array}$ & $\begin{array}{c}\text { Cylindrocladium } \\
\text { sp. }\end{array}$ \\
\hline Americanas & 84 & 10,28 & 16,82 & 12,14 & 11,21 & 18,69 & 17,75 & 5,60 \\
\hline Bordô & 29 & 0,93 & 2,80 & 2,80 & 1,86 & 8,41 & 2,80 & 1,86 \\
\hline Concord & 17 & 1,86 & 5,60 & 1,86 & 4,67 & 3,73 & 3,73 & 0,93 \\
\hline Isabel & 7 & 0,93 & 1,86 & 0 & 0,93 & 0,93 & 0,93 & 0,93 \\
\hline Niágara & 31 & 6,54 & 6,54 & 7,47 & 3,73 & 5,60 & 10,28 & 1,86 \\
\hline Hibridas & 11 & 0,93 & 0,93 & 0 & 2,80 & 0,93 & 1,86 & 1,86 \\
\hline Couderc & 3 & 0 & 0,93 & 0 & 0,93 & 0,93 & 0 & 0 \\
\hline Dona Zilá & 1 & 0 & 0 & 0 & 0,93 & 0 & 0 & 0 \\
\hline Moscato-Embrapa & 3 & 0 & 0 & 0 & 0,93 & 0 & 0,93 & 0 \\
\hline Seibel & 1 & 0 & 0 & 0 & 0 & 0 & 0,93 & 0 \\
\hline Tardia de Caxias & 2 & 0 & 0 & 0 & 0 & 0 & 0 & 2 \\
\hline Vênus & 1 & 0,93 & 0 & 0 & 0 & 0 & 0 & 0 \\
\hline Viníferas & 12 & 1,86 & 3,73 & 1,86 & 2,80 & 0 & 2,80 & 0 \\
\hline Cabernet Sauvignon & 2 & 0,93 & 1,86 & 0,93 & 0 & 0 & 0 & 0 \\
\hline Cabernet Franc & 3 & 0 & 0 & 0,93 & 0,93 & 0 & 0 & 0 \\
\hline Moscato-Yalo & 2 & 0 & 0,93 & 0 & 0,93 & 0 & 0,93 & 0 \\
\hline Perlona & 1 & 0 & 0 & 0 & 0 & 0 & 0,93 & 0 \\
\hline Riesling Itálico & 1 & 0,93 & 0 & 0 & 0 & 0 & 0,93 & 0 \\
\hline Tannat & 2 & 0 & 0 & 0 & 0,93 & 0 & 0 & 0 \\
\hline Trebbiano & 1 & 0 & 0,93 & 0 & 0 & 0 & 0 & 0 \\
\hline Total & 107 & 13,08 & 21,49 & 14,01 & 16,82 & 19,62 & 22,42 & 7,47 \\
\hline
\end{tabular}




\section{L.R. Garrido et al}

nizam os tecidos internos de videiras são mais ou menos patogênicas, mas a expressão dos sintomas depende de outros fatores, como a idade e suscetibilidade das cultivares infetadas, o modo de infecção e a funcionalidade do vasos invadidos, a reação dos tecidos dos hospedeiros e fatores ambientais como a disponibilidade de nutrientes, água, temperatura, entre outros (Graniti et al., 2000). Inoculações com P. chlamydosporum na cv. 'Chardonnay' mostraram sintomas de redução de crescimento, descoloração dos vasos e declínio após 2,5 anos (Scheck et al., 1998).

O fungo $F$. oxysporum f.sp. herbemontis é nativo da região Sul do Brasil, ocorrendo no Rio Grande do Sul e em Santa Catarina. Em cerca de 12 amostras de uvas americanas e em três amostras de uvas viníferas foi verificada a presença de fusariose. Nas viníferas, a espécie foi constatada no portaenxerto SO4 que é extremamente suscetível a este patógeno. Das cultivares americanas produzidas por estaquia a mais tolerante é a 'Isabel'. Nas cultivares de porta-enxerto 'Paulsen 1103' e 'R99' recomendadas há alguns anos, na região, não tem sido constadada a ocorrência da fusariose.

Uma menor incidência de declínio e morte de videiras foi verificada nas uvas híbridas e viníferas. Embora o número de amostras das cultivares pertencentes a estes grupos seja significativamente menor do que o grupo das uvas americanas, o levantamento foi realizado tendo por base informações dos técnicos locais dos municípios visitados, ou seja, não houve uma intenção de coletar o mesmo número de amostras em vinhedos de cultivares $V$. vinifera e $V$. labrusca.

Uma maior incidência de fungos, já relatados anteriormente, foi observada em plantas propagadas por estaquia $(50,4 \%)$ pé-franco, do que em plantas enxertadas (11,2\%). Estas diferenças são atribuídas, em parte, a melhor sanidade das mudas enxertadas, muitas delas importadas. Também é importante salientar que a ocorrência de um determinado patógeno, como por exemplo, F. oxysporum f.sp. herbemontis, não impede o aparecimento de outras espécies de fungos nas amostras, tendo em vista que as mesmas foram coletadas a campo. Assim, o declínio observado em algumas amostras de videira pode ser resultado de mais de um fator biótico e sua interação com os fatores abióticos existentes. De um modo geral, não se observou uma grande diferença na ocorrência de fungos, que infetam o sistema radicular, comparado aos da parte aérea (Tabela 1), embora espécies patogênicas, do primeiro grupo, sejam mais difíceis de controlar na ausência de porta-enxertos resistentes.

Das amostras de porta-enxertos identificados, observou-se a presença de Cylindrocarpon sp. (33,3\%), e Verticillium sp. (50\%) no 'Paulsen 1103', Cylindrocarpon sp. (100\%) no '101-14', e F. oxysporum f.sp. herbemontis (100\%) no 'SO4'. A inoculação de plantas de 'Bordô' com um isolado de Cylindrocarpon sp. confirmou a patogenicidade e os mesmos resultados foram observados na avaliação da resistência de alguns porta-enxertos e cultivares de videira (dados não mostrados). Resultados similares foram encontrados por Rego et al. (2000) após a inoculação de Cylindrocarpon destructans em diferentes cultivares de porta-enxertos, confirmando a suscetibilidade da maioria deles.

\section{REFERÊNCIAS BIBLIOGRÁFICAS}

AUGer, J., ESterio, M., CASTILlo, G., DROGUETT, A., VENEGAS, J. \& REVECO, M. Propagación de plantas de vid (Vitis vinifera L.) cv. Red Globe libres de Cylindrocarpon destructans (Zinss.), Scholten. Fitopatologia Brasileira 26:454. 2001.

BARNETT, H.L. \& HUNTER, B.B. Illustrated genera of imperfect fungi. $3^{\text {a }}$ ed. Minneapolis. Burgess Publishing Company. 1972.

CARMICHAEL, J.W., BRYCEKENDRICK, W., CONNERS, I.L. $\&$ SIGLER, L. Genera of hyphomycetes. Alberta. The University of Alberta Press. 1980.

ELLIS, M.B. Dematiaceous hyphomicetes. Kew. Commonwelth Mycological Institute. 1971.

GARRIDO. L. DA R. \& SÔNEGO, O.R. Chave para identificação de agentes causadores de declínio da videira. Bento Gonçalves: Embrapa Uva e Vinho. Embrapa Uva e Vinho. Circular Técnica, 26. 1999.

GRANITI, A., SURICO, G. \& MUGNAI, L. Esca of grapevine: a disease complex or a complex of diseases? Phytopathologia Mediterranea 39:16-20. 2000.

GRASSO, S. \& MAGNANO DI SAN LIO, G. Infezioni di Cylindrocarpon obtusisporum su piante di vite in Sicilia. Vitis 14:3639. 1975.

HANLIN, R.T. Illustrated genera of ascomycetes. St Paul. APS Press. 1990.

KUHN, G.B. Morte de plantas de videira (Vitis spp.) devido à ocorrência de fungos causadores de podridões radiculares e doenças vasculares. Bento Gonçalves: Embrapa -UEPAE, Embrapa - UEPAE. Circular Técnica, 6. 1981.

MELLO, L.M.R. de. I - Cadastro vitícola. In.: Mello, L.M.R. de. (Ed.). Cadastro vitícola do Rio Grande do Sul - 1995 / 2000. Versão 1.0. Bento Gonçalves: Embrapa Uva e Vinho / Ibravin. 2001. CDROM.

PEARSON, R.C. \& GOHEEN, A.C. Compendium of grape diseases. St. Paul. APS Press. 1988.

REGO, C., Oliveira, H., CARVAlHO, A. \& PHILliPS, A. Involvement of Phaeoacremonium spp. and Cylindrocarpon destructans with grapevine decline in Portugal. Phytopathologia Mediterranea 39:76-79. 2000.

SCHECK, H.J., VASQUEZ, S.J., FOGLE, D. \& GUBLER, W.D. Grape growers report losses to black-food and grapevine decline. California Agriculture 52:19-23. 1998.

SÔNEGO, O.R., INÁCIO, C.A. \& DIANESE, J.C. First report of Botryosphaeria dothidea and Eutypa lata on grapevine in Rio Grande do Sul. Fitopatologia Brasileira 24:333. 1999.

SÔNEGO, O.R., BOTTON, M., MAIA, J.D.G. \& GARRIDO, L. DA. R. III - Doenças e pragas. In.: Maia, J.D.G. \& Kuhn, G.B. (Eds.). Cultivo da Niágara rosada em áreas tropicais do Brasil. Bento Gonçalves: Embrapa Uva e Vinho, 2001. pp.45-63. 